IFIC/07-12

\title{
Integrating out strange quarks in ChPT
}

\author{
J. Gasser ${ }^{\mathrm{a}}$, Ch. Haefeli ${ }^{\mathrm{b}}$, M.A. Ivanov ${ }^{\mathrm{c}}$ and M. Schmid ${ }^{\mathrm{a}}$ \\ ${ }^{a}$ Institute for Theoretical Physics, University of Bern, Sidlerstr. 5, CH-3012 \\ Bern, Switzerland \\ b Departament de Física Teòrica, IFIC, Universitat de València-CSIC, Apt. \\ Correus 22085, E-46071 València, Spain \\ ${ }^{\mathrm{c}}$ Laboratory of Theoretical Physics, Joint Institute for Nuclear Research, \\ 141980 Dubna (Moscow region), Russia
}

\begin{abstract}
We study three flavour chiral perturbation theory in a limit where the strange quark mass is much larger than the external momenta and the up and down quark masses, and where the external fields are those of two-flavour chiral perturbation theory. In this case, the theory reduces to the one of $S U(2)_{L} \times S U(2)_{R}$. Through this reduction, one can work out the strange quark mass dependence of the LECs in the two-flavour case. We present the pertinent relations at two-loop order for $F, B$ and $l_{i}$.
\end{abstract}

Key words: Chiral symmetries, Chiral perturbation theory, Chiral lagrangians PACS: 11.30.Rd, 12.39.Fe, 11.40.Ex

1. We consider Green functions of quark currents in the framework of QCD with three flavours. At low energies, the Green functions can be analysed in the framework of chiral perturbation theory $(\mathrm{ChPT})[1-3]$. It is customary to perform the pertinent quark mass expansion either around $m_{u}=m_{d}=0$, with the strange quark mass held fixed at its physical value $\left(\mathrm{ChPT}_{2}\right)$, or to consider an expansion in all three quark masses, around $m_{u}=m_{d}=m_{s}=0\left(\mathrm{ChPT}_{3}\right)$. The relevant effective lagrangians contain low-energy constants (LECs) which are not determined by chiral symmetry alone. The two expansions are not independent: one can express the LECs in the two-flavour case through the ones in $\mathrm{ChPT}_{3}$. These relations were given at one-loop order in [3] and were used to obtain information on the LECs in $\mathrm{ChPT}_{3}$ from those known in the two-flavour case. Because there are many two-loop calculations available now, both in the two- and three-flavour case [4], it is expedient to have the relevant relations between the LECs at two-loop accuracy as well, both, to obtain more 
additional information, and for internal consistency checks. It is the purpose of this letter to provide the relations that occur at order $p^{2}$ and $p^{4}$ in $\mathrm{ChPT}_{2}$.

We comment on related work that is available in the literature. i) The strange quark mass dependence of the $\mathrm{ChPT}_{2}$ LECs at order $p^{2}, p^{4}$ can be worked out at two-loop order from existing two-loop calculations in the three-flavour sector, see below. ii) The strange quark mass expansion of the $\mathrm{ChPT}_{2} \mathrm{LEC} B$ $\left(F^{2} B\right)$ was already provided at this accuracy in Ref. [5] ([6]). iii) The authors of Refs. $[7,8]$ investigate what happens if chiral symmetry breaking exhibits different patterns in $\mathrm{ChPT}_{2}$ and $\mathrm{ChPT}_{3}$. The literature on the subject may be traced from Ref. [8]. In this scenario, a substantial strange quark mass dependence may show up, as a result of which $\mathrm{ChPT}_{3}$ must be reordered and the effect of vacuum fluctuations of $\bar{s} s$ pairs summed up. Whether the relations provided below favour such a situation is not investigated here - the present work just provides the algebraic dependences of the $\mathrm{ChPT}_{2} \mathrm{LECs}_{\mathrm{C}}$ the strange quark mass, at two-loop order. iv) Analogous work was performed at one-loop accuracy in the baryon sector in Ref. [9], and for electromagnetic corrections in Refs. [10-12].

2. We first illustrate how the relations between the LECs emerge, and consider the pion matrix element of the vector current,

$$
\left\langle\pi^{+}\left(p^{\prime}\right)\left|\frac{1}{2}\left(\bar{u} \gamma_{\mu} u-\bar{d} \gamma_{\mu} d\right)\right| \pi^{+}(p)\right\rangle=\left(p+p^{\prime}\right)_{\mu} F_{V}(t) ; t=\left(p^{\prime}-p\right)^{2},
$$

in the chiral limit $m_{u}=m_{d}=0$. In the three-flavour case, at one-loop order, the result reads in $d$ space-time dimensions

$$
F_{V, 3}(t)=1+\frac{t}{F_{0}^{2}}\left[\Phi(t, 0 ; d)+\frac{1}{2} \Phi\left(t, M_{K} ; d\right)\right]+\frac{2 L_{9} t}{F_{0}^{2}} .
$$

The loop function $\Phi$, generated by pions and kaons running in the loop, is given by

$$
\Phi(t, M ; d)=\frac{\Gamma\left(2-\frac{d}{2}\right)}{2(4 \pi)^{d / 2}} \int_{0}^{1} d u u^{2}\left[M^{2}-\frac{t}{4}\left(1-u^{2}\right)\right]^{\frac{d-4}{2}} .
$$

Furthermore, $F_{0}$ denotes the pion decay constant at $m_{u}=m_{d}=m_{s}=0$, and $L_{9}$ is one of the LECs in $\mathrm{ChPT}_{3}$ at order $p^{4}$.

In $\mathrm{ChPT}_{2}$, the corresponding one-loop expression is

$$
F_{V, 2}(t)=1+\frac{t}{F^{2}} \Phi(t, 0 ; d)-\frac{l_{6} t}{F^{2}},
$$

where $F$ denotes the pion decay constant at $m_{u}=m_{d}=0, m_{s} \neq 0$, and where $l_{6}$ stands for a low-energy constant in $\mathrm{ChPT}_{2}$ at order $p^{4}$. If one identifies $F$ with $F_{0}$ at this order, the expressions $F_{V, 3}$ and $F_{V, 2}$ still differ in the coefficient of the term proportional to $t$, and in the contribution $\Phi\left(t, M_{K} ; d\right)$, which 


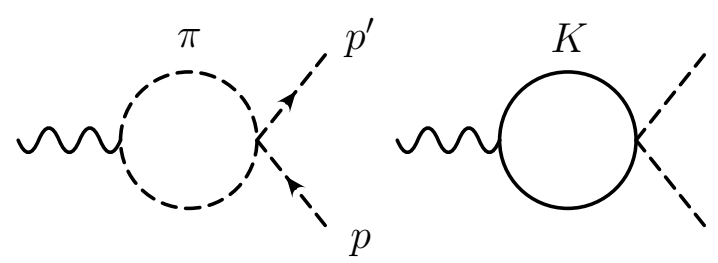

Fig. 1. Loops that generate branch points in the function $\Phi(t, M ; d)$. Dashed (solid) lines denote pions (kaons), the wiggly line is the vector current. Tadpoles are not displayed - these contribute with a constant term to $F_{V}$, as a result of which the form factor is normalised to one at $t=0$, as is required by the pertinent Ward identity.

is absent in the two-flavour case, because kaons are integrated out in that framework.

To proceed, we note that the loop function $\Phi$ is holomorphic in the complex $t$-plane, cut along the real axis for Re $t \geq 4 M^{2}$. We display in Fig. 1 the loops that generate these branch points: pions (kaons) for the one at $t=0(t=$ $\left.4 M_{K}^{2}\right)$. Therefore, $\Phi(t, 0 ; d)$ has a branch point at $t=0$, whereas $\Phi\left(t, M_{K} ; d\right)$ reduces to a polynomial at $t / M_{K}^{2} \ll 1$,

$$
\Phi\left(t, M_{K} ; d\right)=\sum_{l=0}^{\infty} \Phi_{l}\left(M_{K}, d\right)\left(\frac{t}{M_{K}^{2}}\right)^{l} .
$$

Let us discard for a moment the terms of order $t$ and higher in this expansion. It is then seen that $F_{V, 3}$ reduces to $F_{V, 2}$, provided that we set

$$
l_{6}=-2 L_{9}-\frac{1}{2} \Phi_{0}\left(M_{K}, d\right) .
$$

At $d=4$, this relation reduces to the one between the renormalised LECs $l_{6}^{r}$ and $L_{9}^{r}$, provided in [3], see also below.

We conclude that, at low energies, the expression of the vector form factor in $\mathrm{ChPT}_{3}$ reduces to the one in the two-flavour case, up to polynomial terms of order $t^{2}$ and higher, using properly matched LECs in the two-flavour framework. A similar statement holds true for all Green functions of quark currents built from up and down quarks alone, see below.

3. We now come back to the higher-order terms in Eq. (5). We start with the observation that the term of order $t^{l}$ contributes at order $t^{l+1}$ to $F_{V, 2}$ - those with $l \geq 1$ are thus of the same chiral order in $F_{V, 2}$ as the ones generated by graphs with $l+1$ loops in $\mathrm{ChPT}_{2}$. Apparently, one runs into a problem with power counting here: the low-energy expansion of the one-loop contribution in $\mathrm{ChPT}_{3}$ amounts to terms of arbitrarily high orders in the $S U(2) \times S U(2)$ expansion of $F_{V, 2}$. Indeed, this is a rule rather than an exception: Because the strange quark mass is counted as a quantity of chiral order zero in $\mathrm{ChPT}_{2}$, the counting of a quantity like $t / M_{K}^{2}$ is different in the two theories. As a result 
of this, higher-order loops in $\mathrm{ChPT}_{3}$ in general start to contribute already at leading order in $\mathrm{ChPT}_{2}$.

As will be discussed in our forthcoming publication [13], a systematic and coherent scheme is obtained by counting $n$-loop contributions - and, in particular the relevant LECs - to be of order $\hbar^{n}$, and the strange quark mass to be of order $\hbar^{-1}$. In this manner, it is easily seen that e.g. the term with $l=1$ in (5) contributes at order $\hbar^{2}$ to $F_{V, 2}$ and amounts to a contribution from the LECs at two-loop order in $\mathrm{ChPT}_{2}$, etc.

We call in the following the contributions from tree, one-loop and two-loop graphs in $\mathrm{ChPT}_{3}$ to the two-flavour LECs leading, next-to-leading and nextto-next-to-leading order contributions, respectively. The relation for the renormalised LEC $l_{6}^{r}$ is

$$
l_{6}^{r}=\alpha+\beta y+O\left(y^{2}\right), \quad y=\frac{B_{0} m_{s}}{16 \pi^{2} F_{0}^{2}},
$$

with coefficients $\alpha, \beta, \ldots$ that are polynomials in the LECs of $\mathrm{ChPT}_{3}$ and in the logarithms of the meson masses ${ }^{1}$. The quantity $\alpha(\beta y)$ denotes the NLO (NNLO) term, generated by one-loop (two-loop) graphs in $\mathrm{ChPT}_{3}$. They are all of order $\hbar$ according to the above counting rules. [There is no contribution from tree graphs for $l_{6}^{r}$.]

For illustration, we note that from Eq. (6), one finds

$$
\alpha=\frac{1}{192 \pi^{2}}\left[\ln \left(B_{0} m_{s} / \mu^{2}\right)+1\right]-2 L_{9}^{r}(\mu)
$$

where $\mu$ denotes the renormalisation scale.

In conclusion, the above counting of loops and of $m_{s}$ in powers of $\hbar$ generates a systematic (Laurent)-series of the LECs in the variable $y$, modulo logarithmic terms, and greatly simplifies the counting.

4. We now investigate the expansion of the LECs in general, and discuss how one can determine the NNLO terms that we are after here. An obvious procedure is the one used above for $l_{6}^{r}$ : One compares matrix elements, evaluated in $S U(2) \times S U(2)$ with the same ones evaluated in $S U(3) \times S U(3)$. In the case of $F, B, l_{1}, \ldots l_{6}$, available loop calculations allow one to perform the matching at NNLO. We refer to this framework as method $I$ in the following. In order to match the LECs at order $p^{6}$ to the same accuracy in this manner, a

1 We use the following notation. Order $p^{2} ; p^{4}: F, B ; l_{1}, \ldots, l_{7}, h_{1}, h_{2}, h_{3}\left(\mathrm{ChPT}_{2}[2]\right)$; $F_{0}, B_{0} ; L_{1}, \ldots, L_{10}, H_{1}, H_{2}\left(\mathrm{ChPT}_{3}[3]\right)$. Order $p^{6}: C_{1}, \ldots, C_{94}\left(\mathrm{ChPT}_{3}[14,15]\right)$, and similarly for the renormalised quantities $l_{i}^{r}, \ldots, C_{i}^{r}$. 


\begin{tabular}{cccccc}
\hline \hline LEC & Source & Ref. & LEC & Source & Ref. \\
\hline$F$ & $F_{\pi}$ & {$[17]$} & $F^{2} B$ & $\langle 0|\bar{u} u| 0\rangle$ & {$[6,18]$} \\
$l_{1,2}^{r}$ & $\pi \pi \rightarrow \pi \pi$ & {$[19]$} & $l_{3}^{r}$ & $M_{\pi}$ & {$[17]$} \\
$l_{4}^{r}$ & $F_{\pi}, M_{\pi}$ & {$[17]$} & $l_{5}^{r}$ & $\left\langle 0\left|A_{\mu}^{i} A_{\nu}^{k}\right| 0\right\rangle,\left\langle 0\left|V_{\mu}^{i} V_{\nu}^{k}\right| 0\right\rangle$ & {$[17]$} \\
$l_{6}^{r}$ & $F_{V}(t)$ & {$[20]$} & $h_{1}^{r}$ & $\langle 0|\bar{u} u| 0\rangle, M_{\pi}, F_{\pi}$ & {$[17,18]$} \\
$h_{2}^{r}$ & $\left\langle 0\left|V_{\mu}^{i} V_{\nu}^{k}\right| 0\right\rangle$ & {$[17]$} & $h_{3}$ & $\left\langle 0\left|S^{i} S^{k}\right| 0\right\rangle, B$ & {$[17,21]$} \\
\hline \hline
\end{tabular}

Table 1

The quantities used to match $F, B ; l_{i}, h_{i}$ at NNLO (method I). The results for $F, B, l_{1}, \ldots, l_{6}$ and $h_{i}$ agree with method II [13], and the result for $B$ agrees with the calculation performed in [5]. The matching for $l_{7}$ was only performed with method II, see text.

tremendous amount of two-loop calculations in $\mathrm{ChPT}_{2,3}$ would be required. We believe that it is fair to say that these will never be performed.

Therefore, and in order to have an independent check on the results, we have developed $[13,16]$ a generic method $I I$, based on the path integral formulation of ChPT. It consists in the evaluation of the local terms that are generated in the framework of $\mathrm{ChPT}_{3}$ by graphs where heavy particles are running in the loops, and identifying these with local contributions generated by the counterterms in $\mathrm{ChPT}_{2}$. This method allows one to perform the matching for the LECs at order $p^{6}$ as well. Because the technique is rather involved, we defer a detailed description of the framework to a forthcoming publication $[13]$.

We have performed the matching of $F, B ; l_{1}, \ldots, l_{6}, h_{i}$ at NNLO in both frameworks, see table 1 for the quantities invoked in method I. (The analytical formulae for the two-loop $\mathrm{ChPT}_{3}$ quantities are provided in [22], whereas the ones of $\mathrm{ChPT}_{2}$ are only needed at one-loop order.). The results of the two calculations fully agree. This is a highly nontrivial check on our calculation (and on the corresponding two-loop $\mathrm{ChPT}_{3}$ one). As for $l_{7}$, the correlator $\left\langle 0\left|T \bar{u} \gamma_{5} u(x) \bar{u} \gamma_{5} u(0)\right| 0\right\rangle$ might be invoked in method I, in order to check the result obtained with method II. This correlator is, however, not available in the literature to the best of our knowledge. We found that the available two-loop expressions for the neutral pion mass [23] - from where one could determine $l_{7}$ as well in principle - are too voluminous to be used for this purpose. On the other hand, we have checked several expressions in $l_{7}$ by comparing with the corresponding contributions to the two-point function of two pseudoscalar isoscalar densities, using Eq. (12.10) in Ref. [2].

This completes the discussion of the methods used for the determination of the NNLO terms in the $m_{s}$ expansion of the LECs. In the remaining part of this 
letter, we display the NNLO results for the $p^{2}, p^{4}$ LECs, and then illustrate the use of these in one particular application. To keep this article reasonably short, we do not display the expressions for the contact terms $h_{1,2,3}$. They are available from the authors upon request, and will in addition be presented in [13].

5. All the relations may be put in a form similar to (7). To render the formulae more compact, we found it convenient to slightly reorder the expansions, such that they become a series in the quantity $M_{K}^{2}$, which stands for the one-loop expression of the (kaonmass) ${ }^{2}$ in the limit $m_{u}=m_{d}=0$, see e.g. [3]. The result is

$$
\begin{aligned}
& Y=Y_{0}\left[1+a_{Y} x+b_{Y} x^{2}+\mathcal{O}\left(x^{3}\right)\right], \quad Y=F, \Sigma, \\
& l_{i}^{r}=a_{i}+x b_{i}+\mathcal{O}\left(x^{2}\right), i \neq 7, \\
& l_{7}=\frac{F_{0}^{2}}{8 B_{0} m_{s}}+a_{7}+x b_{7}+\mathcal{O}\left(x^{2}\right), \\
& x=\frac{M_{K}^{2}}{N F_{0}^{2}}, \quad N=16 \pi^{2}, \quad \Sigma=F^{2} B, \quad \Sigma_{0}=F_{0}^{2} B_{0} .
\end{aligned}
$$

We denote the contributions proportional to $a_{i}\left(b_{i}\right)$ as NLO (NNLO) terms. Note that $l_{7}$ receives a contribution at leading order (LO) as well, proportional to $m_{s}^{-1}$. The LO and NLO terms were already determined in [3] - for convenience, we reproduce the $a_{i}$ here,

$$
\begin{array}{lc}
a_{F}=-1 / 2 \ell_{K}+8 L_{4}^{r} N, & a_{\Sigma}=-\ell_{K}-2 / 9 \ell_{\eta}+32 L_{6}^{r} N, \\
a_{1}=-1 / 24 \nu_{K}+4 L_{1}^{r}+2 L_{3}, & a_{2}=-1 / 12 \nu_{K}+4 L_{2}^{r}, \\
a_{3}=-1 / 18 \nu_{\eta}-8 L_{4}^{r}-4 L_{5}^{r}+16 L_{6}^{r}+8 L_{8}^{r}, & \\
a_{4}=-1 / 2 \nu_{K}+8 L_{4}^{r}+4 L_{5}^{r}, & a_{5}=1 / 12 \nu_{K}+L_{10}^{r}, \\
a_{6}=1 / 6 \nu_{K}-2 L_{9}^{r}, & \\
a_{7}=-5 / 18 N^{-1}+1 / 2 \nu_{K}+5 / 9 \nu_{\eta}+4 L_{4}^{r}-4 L_{6}^{r}-36 L_{7}-12 L_{8}^{r},
\end{array}
$$

with the abbreviations

$$
\nu_{P}=\frac{1}{2 N}\left(\ell_{P}+1\right), \ell_{P}=\ln \left(M_{P}^{2} / \mu^{2}\right), \quad P=K, \eta .
$$

In analogy to $M_{K}$, the quantity $M_{\eta}$ denotes the eta mass at one-loop order, in the limit $m_{u}=m_{d}=0$ [3].

The NNLO contributions $b_{i}$ are more involved. As alluded above, its depen- 
dence on the strange quark mass only shows up logarithmically

$$
b=p_{0}+p_{1} \ell_{K}+p_{2} \ell_{K}^{2} .
$$

[We suppress the index $i$ of $b_{i}$ for convenience]. The polynomials $p_{j}$ are independent of the strange quark mass and their scale dependence is such that in combination with the logarithms it adds up to the scale independent quantity $b$. This not only allows for a consistency check on our calculations, but moreover offers the opportunity to rewrite $b$ in a particular compact manner,

$$
b=k+p_{2} \ln ^{2}\left(M_{K}^{2} / \Lambda^{2}\right), \quad k=p_{0}-\frac{p_{1}^{2}}{4 p_{2}} .
$$

By construction the squared logarithm is scale independent and so is the combination $k$. The explicit results for the polynomials $p_{j}$ as well as the logarithm $\ln \left(M_{K}^{2} / \Lambda^{2}\right)$ are displayed in Tab.[2-4]. We introduced scale independent LECs $\bar{L}_{i}$,

$$
L_{i}^{r}=\frac{\Gamma_{i}}{2 N}\left(\bar{L}_{i}+\ell_{K}\right)
$$

with $\Gamma_{i}$ the $\beta$ function of $L_{i}^{r}$ [3], as well as abbreviations for the Clausen function evaluated at two different arguments,

$$
\begin{aligned}
\rho_{1} & =\sqrt{2} \mathrm{Cl}_{2}(\arccos (1 / 3)) \cong 1.41602, \quad \rho_{2}=\sqrt{3} \mathrm{Cl}_{2}(\pi / 3) \cong 1.75793 \\
\mathrm{Cl}_{2}(\theta) & =-\frac{1}{2} \int_{0}^{\theta} d \phi \ln \left(4 \sin ^{2} \frac{\phi}{2}\right) .
\end{aligned}
$$

6. As an application, we discuss the strange quark mass dependence of the scale independent LEC $\bar{l}_{2}$,

$$
\bar{l}_{2}=3 N l_{2}^{r}(\mu)-\ln \frac{M_{\pi}^{2}}{\mu^{2}},
$$

with $M_{\pi}=139.57 \mathrm{MeV}$. Considering $\bar{l}_{2}$ is of interest, because in Ref.[24] it has been determined from a dispersive analysis to rather high precision,

$$
\bar{l}_{2}=4.3 \pm 0.1
$$

One then expects that in combination with the formulae presented here, $\bar{l}_{2}$ provides additional constraints on the pertinent combination of three-flavour LECs. This is furthermore supported from the observation that - aside from the combination $2 C_{13}^{r}-C_{11}^{r}$ - only the two three-flavour LECs $L_{2}^{r}$ and $L_{3}$ appear in the analytical expression. According to Ref. [23], these are known rather precisely,

$$
L_{2}^{r}=(+0.73 \pm 0.12) 10^{-3}, \quad L_{3}=(-2.35 \pm 0.37) 10^{-3} .
$$


Here and in the following, the running scale is taken at $\mu=M_{\rho}=770 \mathrm{MeV}$. Further, at the accuracy we are working, we may identify $F_{0}$ with $F_{\pi}=$ $92.4 \mathrm{MeV}$.

We illustrate the strange quark mass dependence of $\bar{l}_{2}$ in Fig.2 (left panel), where $\bar{l}_{2}$ is shown as a function of $M_{K}^{2}$ at $m_{u}=m_{d}=0$ (as introduced above). The dotted line stands for the NLO approximation, and the NNLO result is shown for two choices for $C_{j}^{r}$ : the dashed line displays the case $2 C_{13}^{r}-C_{11}^{r}=0$, while the solid line is worked out at

$$
2 C_{13}^{r}-C_{11}^{r}=0.6 \cdot 10^{-5}
$$

chosen such that at the physical value of the strange quark mass, the LEC $\bar{l}_{2}$ agrees with the measured one, within the uncertainties. [Note that the singular behaviour at $m_{s} \rightarrow 0$, generated by a chiral logarithm, is not in the validity domain of our formulae any more: the expansion performed here requires that all external momenta are much smaller than $m_{s}$. Remarkably, the pertinent logarithm becomes dominant numerically only for very small $m_{s}$.]

We shortly comment on the $C_{11,13}^{r}$ that occur in this application. In Ref. [25], $C_{13}^{r}$ is worked out from an analysis of scalar form factors. While the result is of the order found in (19), its precise value depends considerably on the input used, see table 2 in Ref. [25] for more information. In Ref. [26, table $12]$ estimates for both LECs $C_{11,13}^{r}$ are provided: the authors find that these do not receive a contribution from resonance exchange at leading order in large $N_{C}$ and therefore vanish at this order of accuracy. Because the scale at which this happens is not fixed a priori, that observation is not necessarily in contradiction with the above result [13].

The impact of these LECs on $\bar{l}_{2}$ is rather enhanced at physical strange quark masses. This is illustrated in Fig.2 (right panel). Taking the LECs $L_{j}^{r}$ from Eq. (18) at face value, the window for a possible choice of the $C_{j}^{r}$ is then very narrow to be in agreement with Eq. (17). To pin down the $C_{j}^{r}$ to good precision including an error analysis requires however a more thorough exploration. In particular, one has to take into account that in the fits performed in Ref. [23], an estimate of order $p^{6}$ counterterm contributions was already used. This work is in progress and is deferred to a forthcoming publication [13].

7. In summary, we have worked out the strange quark mass dependence of the two-flavour LECs at order $p^{2}$ and $p^{4}$. The calculation is performed at next-tonext-to leading order in an expansion in the quantity $x=M_{K}^{2} / 16 \pi^{2} F_{0}^{2}$. The result amounts to twelve relations between the LECs in $\mathrm{ChPT}_{2}$ and the ones in $\mathrm{ChPT}_{3}$. Details of the calculation will be given elsewhere [13].

We have illustrated the use of our results in the case of $\bar{l}_{2}$ : its precise knowledge, 

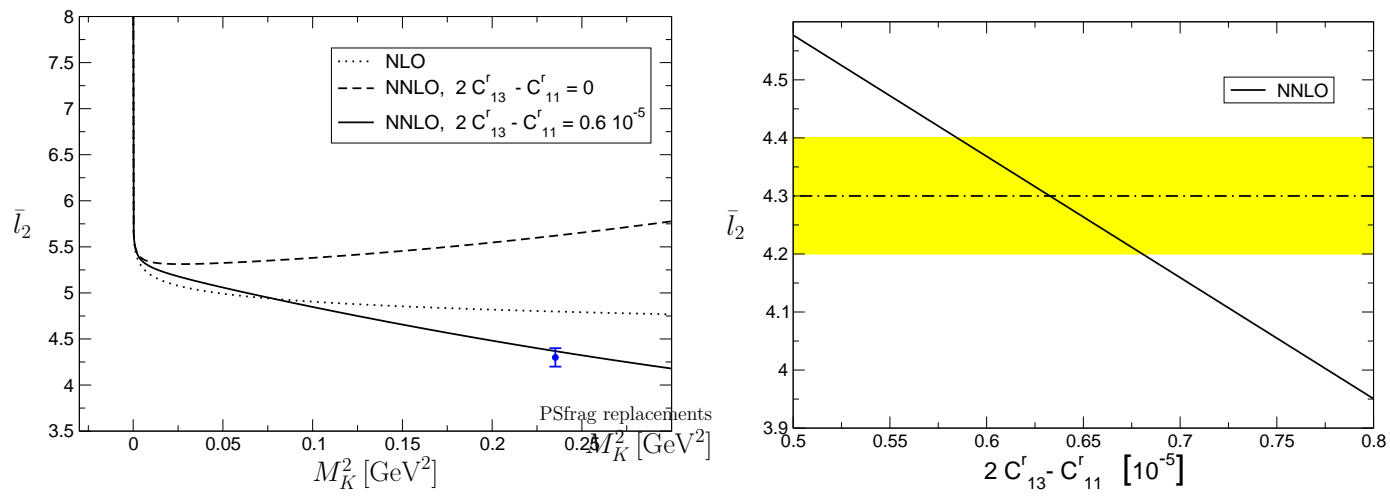

Fig. 2. Left panel: Strange quark mass dependence of $\bar{l}_{2}$. As mentioned in the text, $M_{K}$ denotes the kaon mass at one-loop accuracy in the limit $m_{u}=m_{d}=0$. The physical value of $m_{s}$ corresponds to $M_{K} \approx 485 \mathrm{MeV}$. We show the NLO (dotted line) as well as the NNLO result with two choices for $C_{11,13}^{r}$ : The dashed line corresponds to $2 C_{13}^{r}-C_{11}^{r}=0$, while the solid line is evaluated at (19), which reproduces the prediction from the dispersive analysis [24], Eq. (17) (data point with small error bar). Right panel: Dependence of $\bar{l}_{2}$ on the $p^{6}$ LECs $C_{j}^{r}$ at the physical value of $m_{s}$. The dashed-dotted line with the error band corresponds to the data point and its error bar in the left panel. The running scale is taken at $\mu=M_{\rho}=770 \mathrm{MeV}$.

together with the known values of $L_{2}^{r}, L_{3}$, in principle allow one to pin down the combination $C_{11}-2 C_{13}$ rather precisely. Work on analogous constraints generated by the remaining LECs at order $p^{2}$ and $p^{4}$ is in progress [13]. It will be interesting to merge these constraints with information on the $p^{6}$ LECs from other sources $[4,17,26,27]$.

The strange quark mass dependence of the $S U(2)$ LECs at order $p^{6}$ can be established along similar lines $[13,16]$. The major burden of the calculation is the algebraic complexity of $\mathrm{ChPT}$ at $\mathcal{O}\left(p^{6}\right)$. Various steps and considerable progress towards this goal have already been made [16]. We hope to report on the complete calculation at a later stage.

\begin{tabular}{ll}
\hline \hline & $p_{0}$ \\
\hline$F \quad$ & $-73 / 32+2 / 3 \rho_{1}+1 / 3 \ln \frac{4}{3}+N\left[-52 / 9 L_{2}^{r}-43 / 27 L_{3}\right]$ \\
& $+N^{2}\left[96\left(L_{4}^{r}\right)^{2}+64 L_{4}^{r} L_{5}^{r}-256 L_{4}^{r} L_{6}^{r}-128 L_{4}^{r} L_{8}^{r}+32 C_{16}^{r}\right]$ \\
& $+\ln \frac{4}{3} N\left[128 / 9 L_{1}^{r}+32 / 9 L_{2}^{r}+32 / 9 L_{3}-32 / 3 L_{4}^{r}\right]$ \\
& $26 / 81 \ln ^{2} \frac{4}{3}+N^{2}\left[512 L_{4}^{r} L_{6}^{r}+256 L_{5}^{r} L_{6}^{r}-1024\left(L_{6}^{r}\right)^{2}-512 L_{6}^{r} L_{8}^{r}\right.$ \\
& $\left.+64 C_{20}^{r}+192 C_{21}^{r}\right]+\ln \frac{4}{3} N\left[160 / 9 L_{4}^{r}+16 / 3 L_{5}^{r}-448 / 9 L_{6}^{r}+64 / 3 L_{7}\right.$ \\
\hline \hline
\end{tabular}

Table 2: The polynomial $p_{0}$ defined in Eq.(12) for $F, \Sigma, l_{1}^{r} \ldots l_{6}^{r}, l_{7}$. 


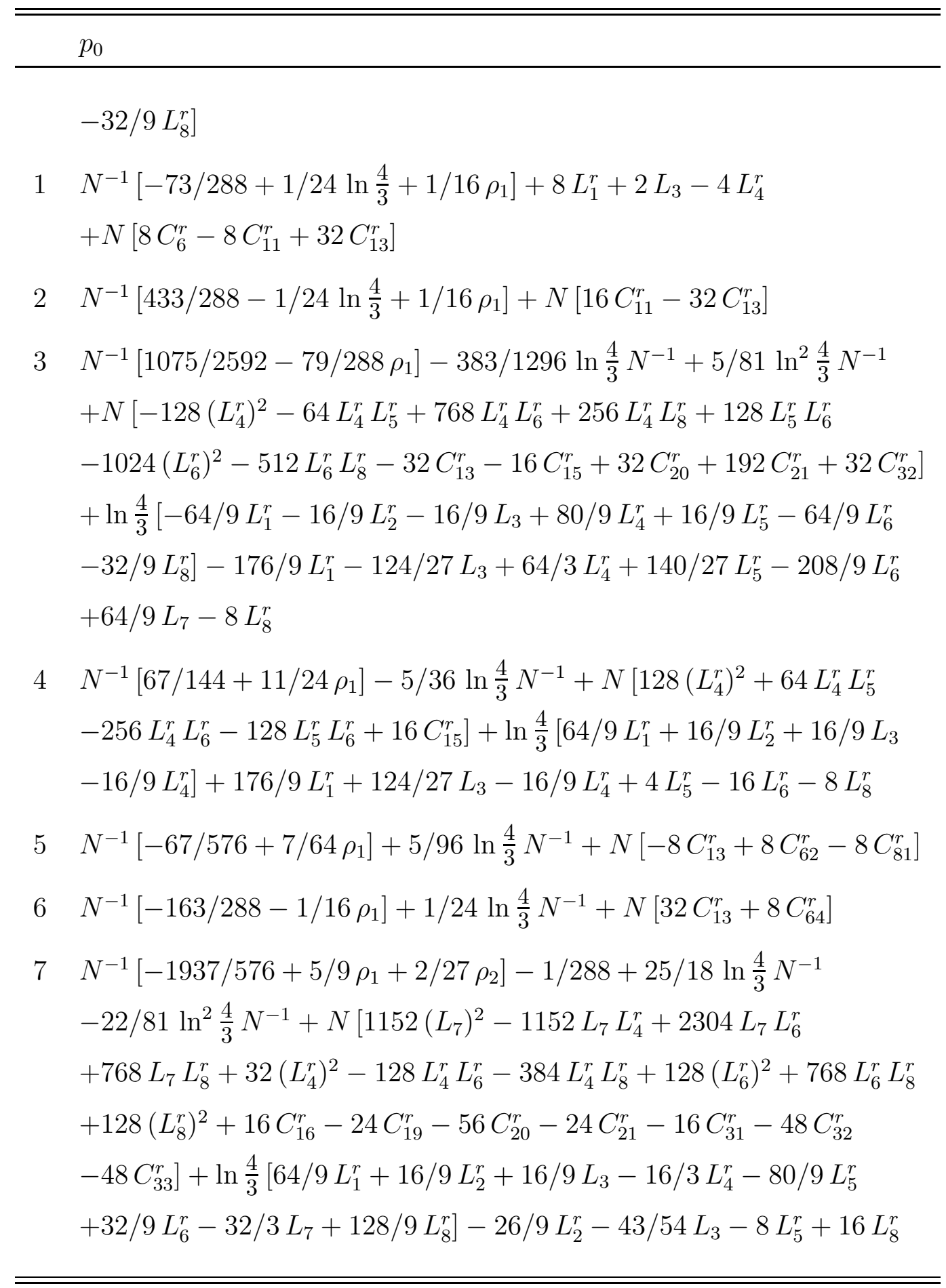

Table 2: The polynomial $p_{0}$ defined in Eq.(12) for $F, \Sigma, l_{1}^{r} \ldots l_{6}^{r}, l_{7}$. 


\begin{tabular}{ll}
\hline \hline & $p_{1}$ \\
\hline$F$ & $7 / 3-1 / 3 \ln \frac{4}{3}+N\left[416 / 9 L_{1}^{r}+104 / 9 L_{2}^{r}+122 / 9 L_{3}-68 / 3 L_{4}^{r}\right]$ \\
$\Sigma$ & $-2 / 81 \ln \frac{4}{3}+N\left[592 / 9 L_{4}^{r}+64 / 3 L_{5}^{r}-1312 / 9 L_{6}^{r}+64 / 3 L_{7}-320 / 9 L_{8}^{r}\right]$ \\
1 & $5 / 6 N^{-1}+8 L_{1}^{r}+4 L_{2}^{r}+3 L_{3}-4 L_{4}^{r}$ \\
2 & $13 / 24 N^{-1}-8 L_{2}^{r}-2 L_{3}$ \\
3 & $-1501 / 648 N^{-1}+11 / 162 \ln \frac{4}{3} N^{-1}-352 / 9 L_{1}^{r}-88 / 9 L_{2}^{r}-106 / 9 L_{3}$ \\
& $+440 / 9 L_{4}^{r}+124 / 9 L_{5}^{r}-496 / 9 L_{6}^{r}-248 / 9 L_{8}^{r}$ \\
4 & $37 / 18 N^{-1}-7 / 18 \ln \frac{4}{3} N^{-1}+352 / 9 L_{1}^{r}+88 / 9 L_{2}^{r}+106 / 9 L_{3}-88 / 9 L_{4}^{r}$ \\
& $-16 L_{6}^{r}-8 L_{8}^{r}$ \\
5 & $17 / 48 N^{-1}-L_{9}^{r}-2 L_{10}^{r}$ \\
6 & $-7 / 24 N^{-1}+2 L_{3}+2 L_{9}^{r}$ \\
7 & $73 / 18 N^{-1}+101 / 162 \ln \frac{4}{3} N^{-1}+208 / 9 L_{1}^{r}+52 / 9 L_{2}^{r}+61 / 9 L_{3}$ \\
& $-52 / 3 L_{4}^{r}-224 / 9 L_{5}^{r}+104 / 9 L_{6}^{r}+184 / 3 L_{7}+632 / 9 L_{8}^{r}$ \\
\hline \hline
\end{tabular}

Table 3: The polynomial $p_{1}$ defined in Eq.(12) for $F, \Sigma, l_{1}^{r} \ldots l_{6}^{r}, l_{7}$.

\begin{tabular}{lll}
\hline \hline \multicolumn{2}{l}{$N p_{2}$} & $\ln \left(M_{K}^{2} / \Lambda^{2}\right)$ \\
\hline$F$ & $-11 / 12 N$ & $-14 / 11+2 / 11 \ln \frac{4}{3}-13 / 11 \bar{L}_{1}-13 / 22 \bar{L}_{2}-244 / 33 L_{3} N$ \\
& & $+17 / 22 \bar{L}_{4}$ \\
$\Sigma$ & $-28 / 81 N$ & $1 / 28 \ln \frac{4}{3}-333 / 56 \bar{L}_{4}-81 / 14 \bar{L}_{5}+451 / 56 \bar{L}_{6}$ \\
& & $-216 / 7 L_{7} N+75 / 28 \bar{L}_{8}$ \\
1 & $-1 / 4$ & $-5 / 3-6 L_{3} N-3 / 4 \bar{L}_{1}-3 / 4 \bar{L}_{2}+1 / 2 \bar{L}_{4}$ \\
2 & $3 / 8$ & $13 / 18-\bar{L}_{2}-8 / 3 L_{3} N$ \\
\hline \hline
\end{tabular}

Table 4: The coefficient $p_{2}$ from Eq.(12) as well as the logarithm defined in Eq.(13) for $F, \Sigma, l_{1}^{r} \ldots l_{6}^{r}, l_{7}$. 


\begin{tabular}{lll}
\hline \hline \multicolumn{1}{l}{$N p_{2}$} & $\ln \left(M_{K}^{2} / \Lambda^{2}\right)$ \\
\hline 3 & $211 / 648$ & $-1501 / 422+22 / 211 \ln \frac{4}{3}-594 / 211 \bar{L}_{1}-297 / 211 \bar{L}_{2}$ \\
& & $-3816 / 211 L_{3} N+990 / 211 \bar{L}_{4}+837 / 211 \bar{L}_{5}-682 / 211 \bar{L}_{6}$ \\
& & $-465 / 211 \bar{L}_{8}$ \\
4 & $-5 / 9$ & $-37 / 20+7 / 20 \ln \frac{4}{3}-33 / 20 \bar{L}_{1}-33 / 40 \bar{L}_{2}-53 / 5 L_{3} N$ \\
& & $+11 / 20 \bar{L}_{4}+11 / 20 \bar{L}_{6}+3 / 8 \bar{L}_{8}$ \\
5 & $-1 / 16$ & $-17 / 6+\bar{L}_{9}-2 \bar{L}_{10}$ \\
6 & $-1 / 8$ & $7 / 6-8 L_{3} N-\bar{L}_{9}$ \\
7 & $17 / 1296$ & $2628 / 17+404 / 17 \ln \frac{4}{3}+702 / 17 \bar{L}_{1}+351 / 17 \bar{L}_{2}$ \\
& & $+4392 / 17 L_{3} N-702 / 17 \bar{L}_{4}-3024 / 17 \bar{L}_{5}+286 / 17 \bar{L}_{6}$ \\
& & $+39744 / 17 L_{7} N+2370 / 17 \bar{L}_{8}$
\end{tabular}

Table 4: The coefficient $p_{2}$ from Eq.(12) as well as the logarithm defined in Eq.(13) for $F, \Sigma, l_{1}^{r} \ldots l_{6}^{r}, l_{7}$.

\section{Acknowledgements.}

The support of Hans Bijnens and Gerhard Ecker was very essential for this work. Hans Bijnens made available to us many of the two-loop calculations performed by him and his collaborators, and even calculated a particular one for our use. Gerhard Ecker provided us with a Form program which simplified the evaluation of the two-loop functional considerably. We are grateful for many useful discussions with Roberto Bonciani, Gilberto Colangelo, Sébastien Descotes-Genon, Andrey I. Davydychev, Thorsten Ewerth, Roland Kaiser, Heiri Leutwyler and Jan Stern, and thank Hans Bijnens, Gilberto Colangelo and Gerhard Ecker for useful comments concerning the manuscript. The calculations were performed partly with Form 3.1 [28]. This work was supported by the Swiss National Science Foundation, by RTN, BBW-Contract No. 01.0357, and EC-Contract HPRN-CT2002-00311 (EURIDICE), by the Ministerio de Educación y Ciencia under the project FPA2004-00996, by Generalitat Valenciana GVACOMP2007-156, and by EU MRTN-CT-2006-035482 (FLAVIAnet). 


\section{References}

[1] S. Weinberg, Physica A 96 (1979) 327.

[2] J. Gasser and H. Leutwyler, Annals Phys. 158 (1984) 142.

[3] J. Gasser and H. Leutwyler, Nucl. Phys. B 250 (1985) 465.

[4] J. Bijnens, Prog. Part. Nucl. Phys. 58, 521 (2007) [hep-ph/0604043].

[5] R. Kaiser and J. Schweizer, JHEP 0606, 009 (2006) [hep-ph/0603153].

[6] B. Moussallam, JHEP 0008, 005 (2000) [hep-ph/0005245].

[7] S. Descotes-Genon, L. Girlanda and J. Stern, JHEP 0001, 041 (2000) [hepph/9910537]; S. Descotes-Genon, L. Girlanda and J. Stern, Eur. Phys. J. C 27, 115 (2003) [hep-ph/0207337]; S. Descotes-Genon, N. H. Fuchs, L. Girlanda and J. Stern, Eur. Phys. J. C 34, 201 (2004) [hep-ph/0311120].

[8] S. Descotes-Genon, hep-ph/0703154.

[9] M. Frink and U. G. Meissner, JHEP 0407, 028 (2004) [hep-lat/0404018].

[10] J. Gasser, V. E. Lyubovitskij, A. Rusetsky and A. Gall, Phys. Rev. D 64 (2001) 016008 [hep-ph/0103157].

[11] H. Jallouli and H. Sazdjian, Phys. Rev. D 58, 014011 (1998) [Erratum-ibid. D 58, 099901 (1998)] [hep-ph/9706450].

[12] A. Nehme, La Brisure d'Isospin dans les Interactions Meson-Meson à Basse Energie, PhD thesis, CPT Marseille, July 2002.

[13] J. Gasser, Ch. Haefeli, M. A. Ivanov and M. Schmid, in preparation.

[14] J. Bijnens, G. Colangelo and G. Ecker, JHEP 9902 (1999) 020 [hepph/9902437].

[15] J. Bijnens, G. Colangelo and G. Ecker, Annals Phys. 280 (2000) 100 [hepph/9907333].

[16] M. Schmid, Strangeless $\chi P T$ at large $m_{s}, \mathrm{PhD}$ thesis, University of Bern, January 2007.

[17] G. Amoros, J. Bijnens and P. Talavera, Nucl. Phys. B 568, 319 (2000) [hep$\mathrm{ph} / 9907264]$.

[18] J. Bijnens and K. Ghorbani, Phys. Lett. B 636, 51 (2006) [hep-lat/0602019].

[19] J. Bijnens, P. Dhonte and P. Talavera, JHEP 0401, 050 (2004) [hepph/0401039].

[20] J. Bijnens and P. Talavera, JHEP 0203, 046 (2002) [hep-ph/0203049].

[21] J. Bijnens, private communication. 
[22] J. Bijnens, http://www.thep.lu.se/ bijnens/chpt.html.

[23] G. Amoros, J. Bijnens and P. Talavera, Nucl. Phys. B 602, 87 (2001) [hep$\mathrm{ph} / 0101127]$.

[24] G. Colangelo, J. Gasser and H. Leutwyler, Nucl. Phys. B 603, 125 (2001) [hep$\mathrm{ph} / 0103088]$.

[25] J. Bijnens and P. Dhonte, JHEP 0310, 061 (2003) [hep-ph/0307044].

[26] V. Cirigliano, G. Ecker, M. Eidemuller, R. Kaiser, A. Pich and J. Portoles, Nucl. Phys. B 753, 139 (2006) [hep-ph/0603205];

[27] B. Moussallam, Nucl. Phys. B 504, 381 (1997) [hep-ph/9701400]; M. Knecht and A. Nyffeler, Eur. Phys. J. C 21, 659 (2001) [hep-ph/0106034]; J. Bijnens and P. Talavera, Nucl. Phys. B 669, 341 (2003) [hep-ph/0303103]; J. Bijnens, E. Gamiz, E. Lipartia and J. Prades, JHEP 0304, 055 (2003) [hep-ph/0304222]; M. Jamin, J. A. Oller and A. Pich, JHEP 0402, 047 (2004) [hep-ph/0401080]; V. Cirigliano, G. Ecker, M. Eidemuller, A. Pich and J. Portoles, Phys. Lett. B 596, 96 (2004) [hep-ph/0404004]; V. Cirigliano, G. Ecker, M. Eidemuller, R. Kaiser, A. Pich and J. Portoles, JHEP 0504, 006 (2005) [hep-ph/0503108]; K. Kampf and B. Moussallam, Eur. Phys. J. C 47, 723 (2006) [hep-ph/0604125].

[28] J. A. M. Vermaseren, math-ph/0010025. 